TRIAL WATCH

\title{
Self-inactivating gene-therapy vector alleviates safety concerns
}

The development of gene therapy has been hampered by safety issues, such as the risk of oncogenic insertion of the vectors used to deliver the therapeutic gene. A new trial could help put gene therapy back on track, as it showed that a self-inactivating (SIN) $\gamma$-retrovirus-based therapy can restore immunity in patients with $\mathrm{X}$-linked severe combined immunodeficiency (X-SCID), and that no vector-induced leukaemias were observed in these individuals after a median follow-up of 33 months (N. Engl. J. Med. 371, 1407-1417; 2014).

Individuals with X-SCID, which is caused by mutations in the gene encoding the interleukin- 2 receptor $\gamma$-chain (IL2RG), are at high risk of fatal infections early in life unless they receive a haematopoietic stem cell transplantation (HSCT), ideally from a matched donor. At the beginning of this century, trials of first-generation gene therapy that used $\gamma$-retroviral vectors to deliver IL2RG successfully restored immunity in individuals with X-SCID. Unfortunately, insertion of the vector upstream of oncogenes increased oncogene expression, leading to the clonal expansion of malignant cells and T-cell acute lymphoblastic leukaemia in $25 \%$ of patients, with a median latency of 33 months.

In the SIN $\gamma$-retroviral vector, the transcription-inducing $U 3$ region of the vector long terminal repeat was deleted and IL2RG transcription was driven by an internal promoter derived from the human eukaryotic elongation factor 1 a gene, which is less efficient than U3 at driving gene expression. "The rationale was that by eliminating the enhancer activity, but still having a promoter activity to get transcription, we could get efficacy without toxicity," explains Alain Fischer, a professor at Collège de France and Director of Imagine Institute, France, who was one of the lead investigators in the study.

Eight of the nine patients enrolled in the trial survived, and immunity was restored in seven of these patients. As a molecular surrogate for long-term safety, the investigators examined the reconstituted T cells from patients up to 35 months after therapy. Although the global distribution of integration sites in patients treated with the original $\gamma$-retroviral vector was similar to that in those treated with the SIN vector, there was no evidence of clonal $\mathrm{T}$ cell expansion in patients treated with the SIN vector. "This indicates that even if insertions near oncogenes occurred, they weren't selected in vivo and did not lead to activation of the oncogenes," says Luigi Naldini, Director of the San Raffaele Telethon Institute for Gene Therapy, Milan, Italy. "I think this is very good evidence that there had been a gain in safety by the vector design."

Other second-generation vectors, particularly SIN lentiviral vectors (which have higher transduction efficacy than do $\gamma$-retroviral vectors), have been used for gene therapy for diseases including WiskottAldrich syndrome, X-linked adrenoleukodystrophy and metachromatic leukodystrophy. "If you put together the data from the different trials it is very interesting to observe that none of the patients have developed leukaemia, and about 30 patients have been treated," says Fischer. "There is obviously an improvement. SIN vectors are safer; of course to know exactly how much safer they are we need more patients and more time."

"The field of gene therapy is finally taking a more positive track," agrees Naldini. Given the high rate of complications with unmatched allogeneic HSCTs, he believes that gene therapy could eventually become the first-line therapy for individuals who have X-SCID but no matched donor. Megan Cully 